\title{
Persistent Post-Surgical Pain after Total Knee Arthroplasty at a Tertiary Care Hospital of a Low-Middle Income Country
}

\author{
Aliya Ahmed* (), Gauhar Afshan, Robyna Khan \\ Department of Anaesthesiology, Aga Khan University Karachi, Pakistan \\ Email: *aliya.ahmed@aku.edu
}

How to cite this paper: Ahmed, A., Afshan, G. and Khan, R. (2022) Persistent Post-Surgical Pain after Total Knee Arthroplasty at a Tertiary Care Hospital of a Low-Middle Income Country. Open Journal of Anesthesiology, 12, 20-33.

https://doi.org/10.4236/ojanes.2022.121003

Received: November 29, 2021

Accepted: January 14, 2022

Published: January 17, 2022

Copyright $\odot 2022$ by author(s) and Scientific Research Publishing Inc. This work is licensed under the Creative Commons Attribution International License (CC BY 4.0).

http://creativecommons.org/licenses/by/4.0/

\begin{abstract}
Background: Persistent post-surgical pain (PPSP) is pain that lasts for 3 months or more after a surgical intervention, where other causes of pain have been excluded. There is scarce knowledge about the prevalence of PPSP in low- and middle-income countries (LMICs). The aim of our study was to assess the prevalence of PPSP after total knee arthroplasty (TKA) at our university hospital and explore factors associated with it. Methods: It was a prospective cross-sectional study. Approval was obtained from the Ethics Review Committee. Patients undergoing elective unilateral total knee arthroplasty under general or regional anaesthesia were recruited over a six months period. A designated pain nurse called the patients three months after surgery and asked about the presence of pain, its location, type, degree and associated factors. Patients who reported pain at three months were called a year after the surgery and the same questions were asked. Results: Eighty-two patients had TKA during the study period. At the time of discharge, 57 (69.5\%) patients were satisfied with their postoperative pain management. Three months after the surgery, 13 (15.8\%) patients reported pain. Pain was mild in 11 and moderate in two patients. At one-year follow-up, three patients (3.6\%) reported pain that was mild to moderate in intensity. Pain disturbed sleep in all three patients and disturbed daily life routines in one patient. No significant difference was found in any of the variables when compared with patients who did not report pain at three months. Conclusions: Although PPSP is a recognized adverse outcome after TKA, little is known about its prevalence in LMICs. In our patient population, $15.8 \%$ reported pain three months after TKA, while at one year, $3.6 \%$ of patients reported mild to moderate pain. Multicenter studies are recommended for determining the overall prevalence in our patient population and for getting directions for making targeted efforts towards its prevention and treatment.
\end{abstract}




\section{Keywords}

Total Knee Arthroplasty, Persistent Postsurgical Pain, Postoperative Pain

\section{Introduction}

Post-surgical pain is one of the top-most fears of patients undergoing surgery. Unrelieved post-surgical pain can have adverse physiological effects and increase morbidity [1]. If this pain continues for an indefinite period, it can have marked negative effects on the patient's quality of life with adverse psychological and social effects and increased financial burden [1] [2]. Persistent post-surgical pain (PPSP), also called chronic post-surgical pain, is defined as pain that develops after a surgical intervention and persists for 3 months or more, where other causes of pain have been excluded [2] [3] [4] [5]. In recent years several studies have shown that there is a high prevalence of prolonged pain after many surgical procedures [6] [7] [8] [9] [10]. However, little is known about the prevalence of PPSP in low- and middle-income countries (LMICs). Research exploring the prevalence, character and factors associated with PPSP following different surgical procedures is essential as it will help us in understanding its burden and identifying the possible contributing factors, which in turn, would guide us in making preventive strategies.

With focused efforts on promoting safe surgery and anaesthesia in LMICs in recent years, the number and complexity of surgeries are on the increase in most LMICs [11]. With this increasing number, the frequency of patients presenting with PPSP is also likely to increase [11]. Total knee arthroplasty (TKA) is one such surgical procedure that is being conducted with increasing frequency in LMICs. Data from developed countries show that most patients undergoing TKA report improvement in pain and function over the weeks following surgery, however, a sizable number may experience PPSP [12]. It is important to assess the prevalence of persistent pain following TKA, to identify the patients at risk and to take measures in the perioperative period to prevent PPSP. Further research will guide us in making strategies for improving patient care and outcomes following TKA [13].

With this perspective, the aim of our study was to assess the prevalence of persistent post-surgical pain after total knee arthroplasty (TKA) performed at our university teaching hospital and explore the factors associated with it.

\section{Methods}

It was a prospective cross-sectional study. Approval was obtained from the institution's Ethics Review Committee (ERC). The study was conducted at the Aga Khan University Hospital, Karachi from June 2014 to December 2015. Patients were recruited at the time of admission for surgery. Written informed consent was taken from each patient and approval was obtained for administration of a 
telephonic questionnaire three months and one year after the surgery. Pain assessment method using verbal numeric rating scale (NRS) of 0 - 10, where 0 referred to no pain and 10 referred to worst imaginable pain, was explained to the patients by the pain nurse. Patients' contact numbers and information about a suitable time to call them were obtained at the time of recruitment. All patients undergoing elective unilateral total knee replacement under general or regional anaesthesia during the six-month study period were included. A formal sample size assessment was not performed. Patients having a revision TKR or those with a history of chronic pain at other sites or taking regular analgesics were excluded, as were those with a known history of psychiatric problems or those having a language barrier.

Choice of anaesthesia was left at the discretion of the primary anaesthesiologist responsible for the anaesthetic management. Standard anaesthetic management protocols were followed for the surgeries conducted under general or regional anaesthesia and no intervention was employed for the purpose of the research. At our hospital, general anaesthesia is induced with propofol $2-3 \mathrm{mg} / \mathrm{kg}$ and cisatracurium $0.15 \mathrm{mg} / \mathrm{kg}$ and is maintained with oxygen, nitrous oxide (40\%:60\%) and isoflurane adjusted to keep a minimum alveolar concentration of 1. Combined spinal epidural (CSE) is employed when surgery is performed under regional anaesthesia. The choice of intraoperative and postoperative analgesic strategies was left at the discretion of the anaesthetic team.

Patients' demographics, intraoperative analgesia used, pain scores at time of discharge from the post-anaesthesia care unit (PACU), postoperative analgesic modalities used and whether acute pain service (APS) was involved in postoperative pain management or not were noted in the data collection form (see appendix). A designated pain nurse asked the patients about their satisfaction with the overall pain management on the day of discharge. The pain nurse noted the date of surgery for each patient, called the patient three months after surgery and filled out the data collection form, including questions on presence of pain, its location, type, degree and associated factors. Patients who reported pain at three months following TKA were followed up again by the pain nurse a year after the surgery, asking the same questions again.

Statistical analysis was performed using statistical packages for social science version 19 (SPSS Inc., Chicago, IL). Persistent postoperative pain and associated factors were the main outcome variables. Quantitative variables were computed by mean and standard deviation and frequency and percentages were computed for qualitative observation.

\section{Results}

Unilateral total knee arthroplasty was performed in 82 patients during the six-month recruitment period. Sixty (73.8\%) of these patients were female and $22(26.8 \%)$ were male. The demographic characteristics, average duration of surgery, type of anaesthesia, intraoperative and postoperative analgesia used and pain score at the time of discharge from PACU are provided in Table 1. Majority 
Table 1. Demographic and perioperative parameters of the study participants.

\begin{tabular}{|c|c|}
\hline \multicolumn{2}{|c|}{$\begin{array}{l}\text { Variables TKA } \\
\qquad \mathrm{N}=82\end{array}$} \\
\hline Age (years) & $62.90 \pm 11.97$ \\
\hline Weight $(\mathrm{kg})$ & $73.77 \pm 13.12$ \\
\hline Duration of surgery (hours) & $4.05 \pm 1.42$ \\
\hline \multicolumn{2}{|l|}{ Anaesthesia type, $\mathrm{n}(\%)$} \\
\hline General Anaesthesia & $33(40.2 \%)$ \\
\hline General Anaesthesia + Epidural & $30(36.6 \%)$ \\
\hline Regional alone & $19(23.2 \%)$ \\
\hline \multicolumn{2}{|l|}{ Intra-operative Analgesia, n (\%) } \\
\hline Epidural & $49(59.7 \%)$ \\
\hline Paracetamol & $44(53.7 \%)$ \\
\hline I. V. Opioids & $24(29.3 \%)$ \\
\hline NSAIDs & $3(3.7 \%)$ \\
\hline \multicolumn{2}{|l|}{ Post-operative Analgesia, n (\%) } \\
\hline Epidural & $49(59.7 \%)$ \\
\hline PCA & $19(23.7 \%)$ \\
\hline Opioid infusion & $5(6.0 \%)$ \\
\hline Intermittent bolus & $5(6.0 \%)$ \\
\hline As required opioids & $4(4.8 \%)$ \\
\hline \multicolumn{2}{|l|}{ Co-Analgesia, n (\%) } \\
\hline Paracetamol & $79(86.3 \%)$ \\
\hline NSAIDs & $11(13.4 \%)$ \\
\hline Tramadol & $5(8.5 \%)$ \\
\hline \multicolumn{2}{|l|}{ Pain at the time of discharge from PACU } \\
\hline Zero & $9(11 \%)$ \\
\hline $1-3$ & $44(53.7 \%)$ \\
\hline $4-6$ & $28(34.1 \%)$ \\
\hline$>6$ & $1(1.2 \%)$ \\
\hline
\end{tabular}

TKA: Total knee arthroplasty; I.V: Intravenous; NSAIDs: Non-steroidal anti-inflammatory drugs; PCA: Patient controlled analgesia; PACU: Post-anaesthesia care unit; APS: Acute Pain Service.

of the patients received general anaesthesia for the surgery. Multimodal analgesia was used for intraoperative and postoperative pain relief in all patients. At the time of discharge from PACU, $65 \%$ of the participants had mild or no pain (Table 1). APS managed the postoperative pain in $69(84.1 \%)$ patients. At the time of discharge from hospital, 57 (69.5\%) patients were satisfied with their postoperative pain management, while $25(30.5 \%)$ gave a neutral response (nei- 
ther satisfied nor dissatisfied).

Three months after the surgery, 13 (15.8\%) patients reported pain. Out of these 13 patients, two were males (15.4\%; $9 \%$ of 22 male patients) and 11 were females (84.6\%; $18 \%$ of the total of 60 female patients). The mean age of the patients with pain at three months was 68.15 years. Pain was mild (NRS 2 - 3) in $84 \%$ of the patients. Pain was mainly aching, burning or stabbing in nature. In six patients, pain occurred only on movement. The type and characteristics of pain are provided in Table 2. Pain disturbed sleep in three patients and affected daily life routines in one patient. Six patients had undergone surgery under general anaesthesia alone with intravenous analgesia, three had received general anaesthesia with epidural infusion for analgesia, while four patients had their surgery under combined spinal epidural (CSE) block. Twelve out of the 13 patients with pain at three months had their postoperative pain managed by APS and were satisfied with their pain management at the time of discharge following TKA. No significant difference was found in any of the above variables when compared with patients who did not report pain at three months. The comparison of these variables among patients who developed pain and those who did not

Table 2. Area, type and characteristics of pain three months after total knee arthroplasty.

\begin{tabular}{|c|c|}
\hline Variables & $\begin{array}{c}\text { TKA } \\
\mathbf{n}=13\end{array}$ \\
\hline \multicolumn{2}{|l|}{ Area of Pain } \\
\hline Site of surgery & $10(76.9 \%)$ \\
\hline Surrounding area & $3(23.1 \%)$ \\
\hline \multicolumn{2}{|l|}{ Type of pain } \\
\hline Aching & $6(46.2 \%)$ \\
\hline Burning & $4(30.8 \%)$ \\
\hline Stabbing & $6(46.2 \%)$ \\
\hline Throbbing & $0(0 \%)$ \\
\hline \multicolumn{2}{|l|}{ Pain score (NRS) } \\
\hline $2-3$ & $11(84.6 \%)$ \\
\hline $4-5$ & $2(15.4 \%)$ \\
\hline \multicolumn{2}{|l|}{ Characteristics of pain } \\
\hline Pain at all time & $1(7.7 \%)$ \\
\hline Limited to a single area & $5(38.5 \%)$ \\
\hline Painful on movement & $6(46.2 \%)$ \\
\hline Altered sensation on touch & $0(0 \%)$ \\
\hline Painful on touch & $1(7.7 \%)$ \\
\hline Painful at rest & $1(7.7 \%)$ \\
\hline No sensation on touch & $0(0 \%)$ \\
\hline
\end{tabular}

NRS: Numeric rating score; TKA: Total knee arthroplasty. 
report pain three months after surgery is provided in Table 3 and Table 4. Patients with pain at 3 months were taking simple over-the-counter analgesics for their pain, including paracetamol and non-steroidal anti-inflammatory agents.

Table 3. Comparison of quantitative factors in patients with persistent pain and those without pain at three months after total knee arthroplasty.

\begin{tabular}{|c|c|c|c|}
\hline \multirow[t]{2}{*}{ Parameters } & $\begin{array}{l}\text { Persistent pain at } \\
3 \text { months } \\
(\mathrm{n}=13)\end{array}$ & $\begin{array}{l}\text { No pain at } \\
3 \text { months } \\
(n=69)\end{array}$ & \multirow[t]{2}{*}{ P-value } \\
\hline & Mean (SD) & Mean (SD) & \\
\hline Age (Years) & $68.15(8.80)$ & $61.91(12.28)$ & 0.085 \\
\hline Weight $(\mathrm{kg})$ & $69.33(9.34)$ & $74.60(13.61)$ & 0.186 \\
\hline Duration of surgery (hour) & $4.00(1.63)$ & $4.06(1.40)$ & 0.885 \\
\hline Pain at discharge from PACU & $3.54(1.19)$ & $2.79(1.61)$ & 0.117 \\
\hline
\end{tabular}

SD: Standard deviation; PACU: Post-anaesthesia care unit.

Table 4. Comparison of factors in patients with persistent post-surgical pain and those without persistent post-surgical pain after total knee arthroplasty.

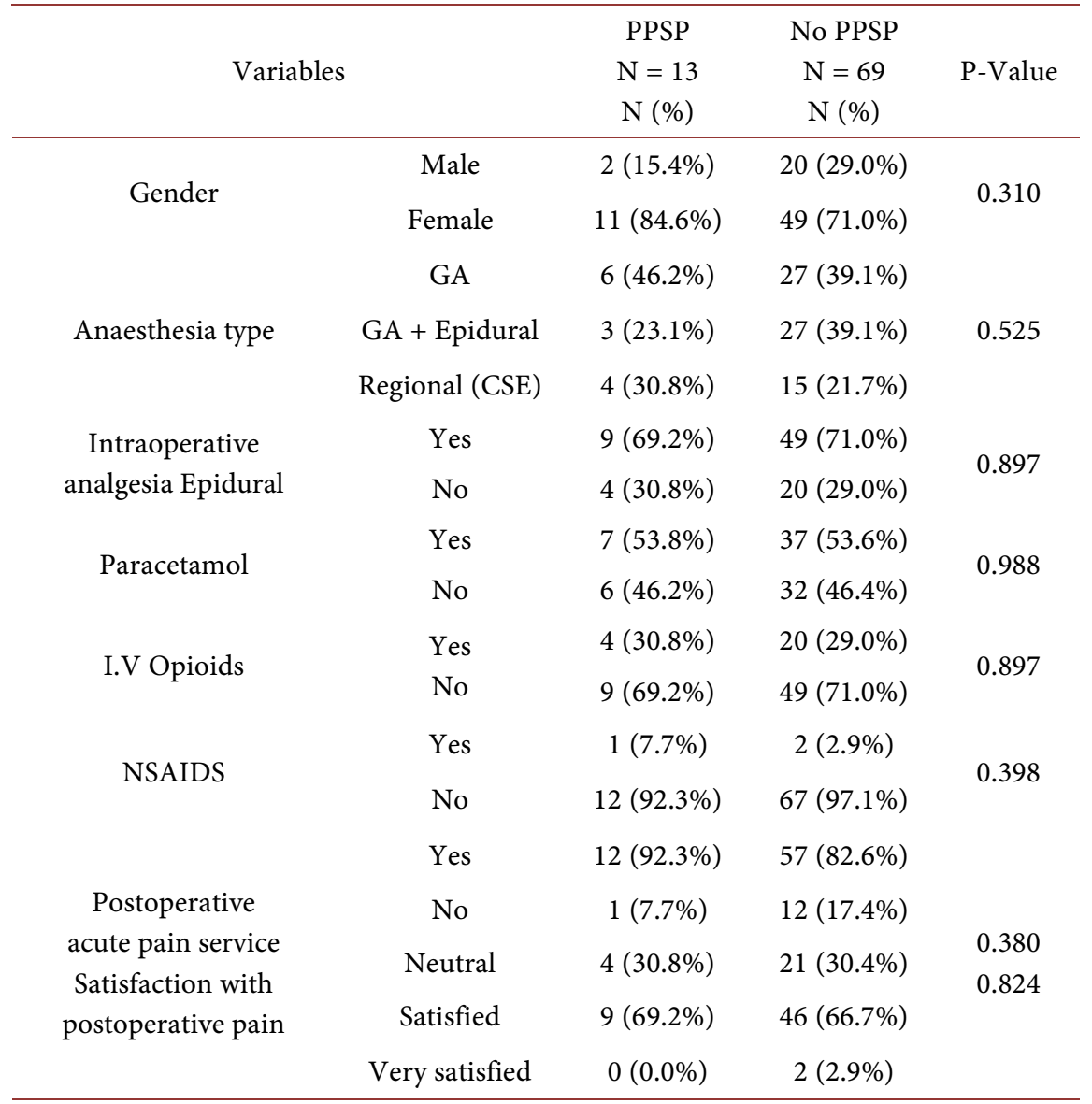

PPSP: Persistent post-surgical pain; GA: General anaesthesia; I.V: Intravenous; NSAIDs: Non-steroidal anti-inflammatory drugs; PCA: Patient controlled analgesia; CSE: combined spinal-epidural. 
At one-year follow-up of the 13 patients who had pain at three months, three (3.6\% of 82 patients who had TKR) reported continued pain, five reported no pain while 4 (30\%) did not respond to our call and one patient had expired due to intracerebral haemorrhage. In the three patients who reported pain, NRS was 3 - 5 and the pain was mostly related to leg movement, especially on standing from a sitting position and walking. Pain disturbed sleep in all three patients and disturbed daily life routines in one patient.

\section{Discussion}

We assessed patients for presence of persistent pain three months and one year following TKA. Our results showed that $73 \%$ of the patients undergoing TKA during the study period were females. Patel et al. [14] in their review of a national database of the United States of America including more than 600 hospitals, reported that out of 262,994 patients undergoing TKA, $61.7 \%$ were females. The likely reason for this finding might be that osteoarthritis is more common among females compared to males [14].

It has been reported that pain severity may plateau at three to six months following TKA [15] and in some patients, pain improvement can continue up to one year after surgery without them eventually ending up with long-term PPSP [16]. However, detection of pain three to six months after TKA can enable the physician to take measures to halt its progression into long-term chronic pain and disability by initiating targeted management for the prevention of PPSP [17]. Werner and Kongsgaard [18] have proposed that pain should be assessed between three and six months following surgery as this would allow adequate time to assess the functional results of the surgery. Appropriate management and encouragement of the patient at this point would help in preventing disappointment with the surgical outcome and the resulting anguish on the part of the patient.

All the 82 patients who participated in the study received multimodal analgesia for postoperative pain relief, which is the recommended strategy [19]. Multimodal analgesia has been shown to provide effective pain relief following TKA and promotes recovery with improved clinical outcomes and better patient satisfaction [19]. Three months following surgery, 13 (15.8\%) patients were found to have pain at the site of incision or immediately surrounding the area. Wylde et al. [20], in their review of chronic pain after TKA, have reported an incidence of persistent pain following TKA of $16 \%$ to $33 \%$. Our results are at the lower end of this range. Pinto et al. found that 39 out of $44(88.6 \%)$ patients included in their study reported pain 4 - 6 months after TKA [21], which is a considerably higher incidence compared to our findings. Singh et al., when comparing studies from 1987 to 1994 with more recent studies, have observed that outcomes of PPSP after TKA have shown improvement over time and the prevalence of PPSP has been decreasing over the past years [22]. However, it is difficult to compare results with other studies because duration and method of follow-up assessment 
varies across studies. Since our results are from a single tertiary care center, they cannot be generalized for the incidence of PPSP following TKA in our patient population.

APS had assessed and managed the postoperative pain of 12 of the 13 patients who reported pain at three months. All of these patients had been satisfied with their postoperative pain management at the time of discharge. It has been seen that lack of knowledge about pain management among healthcare workers, infrequent pain assessments and inadequate treatment of pain are barriers to patient satisfaction with pain management [23]. These deficiencies can be overcome by a well-functioning APS, leading to better satisfaction levels as evidenced in our study, although many of our patients who claimed satisfaction were found to have pain three months after the surgery. Equal number of these patients had received intravenous opioids or epidural local anaesthetic infusion for postoperative pain management, displaying absence of influence of pain management modality on PPSP at three months in our study. However, it has been reported that perioperative epidural analgesia reduces the risk of persistent postsurgical pain [24]. In a meta-analysis, perioperative use of epidural analgesia was shown to reduce persistent post-surgical pain after abdominal surgeries, breast surgeries and thoracotomies [25]. Multicenter studies with larger sample size are required before similar claims could be made for TKA.

Eleven out of the 13 patients with PPSP at three months follow up were females. Singh et al. [22] assessed the impact of gender on pain after TKA and have reported higher pain in women compared to men two years after primary TKA (9\% versus 6.6\%; p 0.04). Ritter et al., in their 5-year follow-up of patients who underwent primary TKA, also reported a difference in pain scores based on gender, women experiencing more pain compared to men [26]. Reasons suggested for this finding are that women have a more advanced disease [27], with worse knee joint function [28] compared to men at the time of surgery. The mean age of the patients complaining of pain at three months was 68.15 years, while mean age of the patients without pain was 61.91 years. Singh et al. [22] have reported lesser pain in older patients compared to patients below 60 years of age two years following primary TKA, providing the possible explanation of less scar formation and increased tolerance for pain in the elderly. On the contrary, Roth et al. [29] have reported no age related differences in pain following TKA.

Pain was aching or burning in nature, or a mixture of these two sensations, but none of our patients complained of numbness or altered sensations at the surgical site. In contrast to our results, Pinto and colleagues found numbness to be present in $77 \%$ of patients who reported pain four to six months after TKA in their study [21]. They found that $46 \%$ of their patients had symptoms that indicated a potential neuropathic element to the pain. Earlier research on different surgical procedures has also claimed that PPSP has a potential neuropathic component [30]. Among our patients, four out of the 13 patients $(30.8 \%)$ had burning pain, but none reported numbness or altered sensations or any other 
indicators of neuropathic pain. We recommend that future studies should include specific questions and indicators during data collection in order to analyze the prevalence of neuropathic pain in patients suffering with PPSP.

All patients who reported pain three months after surgery had mild to moderate pain, the maximum pain score on NRS being 5. Similar pain scores were observed by Pinto et al. following TKA in most of their patients; however $6.8 \%$ of their patients reported severe pain [21]. Other researchers have also found PPSP to be mild to moderate in majority of patients [30] [31]. One of our patients had pain all the time, including at rest, while the others complained of intermittent pain, two to three times a day. Similar findings have been reported for PPSP following TKA [21] [31] by other researchers. Sleep and daily life routines were disturbed due to pain in four patients in our study. This is the most disturbing and feared component of PPSP for the patients and becomes the main reason for their dissatisfaction with surgery. It may lead to depression, low morale and decreased productivity. Preventive strategies with effective perioperative pain control, early detection and prompt treatment of PPSP are important to avoid this outcome [1]. No statistically significant difference was found between patients who reported pain at three months and those who did not with regards to gender, age, weight, type of anaesthesia, postoperative pain management modality, etc. Multicenter trials with a larger sample size are recommended to provide more definitive evidence of the effect of gender and age on PPSP following TKA in our patient population.

At one-year follow-up, out of the 13 patients who had pain three months after TKA, only three (3.6\% of the total 82 patients) reported continued pain. Pain was moderate in severity and disturbed daily routine and mobility. Drosos et al, in their review of studies on prevalence of PPSP after TKA, found an incidence of $13 \%$ to $23 \%$ one-year after TKA [31]. However, they failed to reach firm conclusions about the prevalence of PPSP due to heterogeneity of studies. Under-reporting could well be a reason for difficulty in assessing the true prevalence of PPSP. Wylde and colleagues have reported that a considerable number of patients experiencing constant or daily knee pain do not seek medical help for their pain despite ongoing suffering [20]. Relevant healthcare professionals must seek to understand the reasons behind this attitude and provide guidance to patients regarding the available help and endeavor to improve their management. Better pain relief would improve quality of life, ensure continuity of daily life routines and reduce mental distress on the part of the patient [32]. A multidisciplinary approach is required for effective management of PPSP after TKA, involving family physicians, pain physicians, orthopaedic surgeons, physiotherapists, psychologists, etc.

A limitation of this study is that it is a single center study with a relatively small sample size and no formal estimation of sample size. Pinto and colleagues also reported a single center study with a much smaller sample size of 44 patients [21]. Moreover, at one-year follow-up, there was a $30 \%$ no-response rate, which might have prevented us from getting the true picture of the prevalence of PPSP 
at one year. Also, as this was an initial assessment of PPSP after TKA from our region, we focused mainly on the prevalence and did not assess patients for symptoms indicative of neuropathic pain.

\section{Conclusion}

In conclusion, although PPSP is a recognized adverse outcome after TKA, little is known about its prevalence in LMICs. We have presented the prevalence of PPSP three months and one year after TKA at a university teaching hospital. The prevalence of PPSP following TKA in our study was $15.8 \%$ at three months and its relationship with age and gender was not clearly evident. With global surgery initiatives and a focus on quality and safety in anaesthesia, there is a rise in surgical procedures in LMICs, which is expected to continue in the next decade. Therefore, concurrent research to improve patient outcomes must be a key priority. Multicenter studies are recommended for getting directions for making targeted efforts towards its prevention and treatment. Multicenter studies will also help to determine if there is a difference in prevalence in our patient population compared to data from other parts of the world. Future research should also focus on determining perioperative risk factors for the development of PPSP following TKA. This would help in identifying patients at high risk of PPSP so that preventive strategies could be timely employed.

\section{Conflicts of Interest}

The authors declare no conflicts of interest regarding the publication of this paper.

\section{References}

[1] Gan, T.J. (2017) Poorly Controlled Postoperative Pain: Prevalence, Consequences, and Prevention. Journal of Pain Research, 10, 2287-2298. https://doi.org/10.2147/JPR.S144066

[2] Lavand'Homme, P. and Pogatzki-Zahn, E. (2017) Fact Sheet No. 4: Chronic Postsurgical Pain: Definition, Impact and Prevention. International Association for the Study of Pain.

https://www.europeanpainfederation.eu/wp-content/uploads/2017/01/04.-ChronicPostsurgical-Pain.pdf

[3] Cho, S.H., Kim, Y.R., Lee, J.H. and Kim, S.H. (2012) A Questionnaire Study Investigating the Prevalence of Chronic Postoperative Pain. Korean Journal of Anesthesiology, 62, 40-46. https://doi.org/10.4097/kjae.2012.62.1.40

[4] Macrae, W.A. (2001) Chronic Pain after Surgery. British Journal of Anaesthesia, 87, 88-98. https://doi.org/10.1093/bja/87.1.88

[5] Niraj, G. and Rowbotham, D.J. (2011) Persistent Postoperative Pain: Where Are We Now? British Journal of Anaesthesia, 107, 25-29. https://doi.org/10.1093/bja/aer116

[6] Johansen, A., Romundstad, L., Nielsen, C.S., Schirmer, H. and Stubhaug, A. (2012) Persistent Postsurgical Pain in a General Population: Prevalence and Predictors in the Tromsø Study. Pain, 153, 1390-1396. https://doi.org/10.1016/j.pain.2012.02.018

[7] Gartner, R., Jensen, M.B., Nielsen, J., Edwertz, M., Kroman, N. and Kehlet, H. (2009) 
Prevalence of and Factors Associated with Persistent Pain Following Breast Cancer Surgery. JAMA Network, 302, 1985-1992. https://doi.org/10.1001/jama.2009.1568

[8] Wildgaard, K., Ravn, J. and Kehlet, H. (2009) Chronic Post-Thoracotomy Pain: A Critical Review of Pathogenic Mechanism and Strategies for Prevention. European Journal of Cardio-Thoracic Surgery, 36, 170-180.

https://doi.org/10.1016/j.ejcts.2009.02.005

[9] Brandsborg, B., Nikolajsen, L., Kehlet, H. and Jensen, T.S. (2008) Chronic Pain after Hysterectomy. Acta Anaesthesiologica Scandinavica, 52, 327-331.

https://doi.org/10.1111/j.1399-6576.2007.01552.x

[10] Kehlet, H. (2008) Chronic Pain after Groin Hernia Repair. British Journal of Surgery, 95, 135-136. https://doi.org/10.1002/bjs.6111

[11] Walters, J.L., Jackson, T., Byrne, D. and McQueen, K. (2016) Postsurgical Pain in Low- and Middle-Income Countries. British Journal of Anaesthesia, 116, 153-155. https://doi.org/10.1093/bja/aev449

[12] Lewis, G.N., Rice, D.A., McNair, P.J. and Kluger, M. (2015) Predictors of Persistent Pain after Total Knee Arthroplasty: A Systematic Review and Meta-Analysis. British Journal of Anaesthesia, 114, 551-561. https://doi.org/10.1093/bja/aeu441

[13] Kalso, E.IV. (2013) Persistent Post-Surgery Pain: Research Agenda for Mechanisms, Prevention, and Treatment. British Journal of Anaesthesia, 111, 9-12. https://doi.org/10.1093/bja/aet211

[14] Patel, A.P., Gronbeck, C., Chambers, M., Harrington, M.A. and Halawi, M.J. (2020) Gender and Total Joint Arthroplasty: Variable Outcomes by Procedure Type. Arthroplasty Today, 6, 517-520. https://doi.org/10.1016/j.artd.2020.06.012

[15] Lenguerrand, E., Wylde, V., Gooberman-Hill, R., Sayers, A., Brunton, L., Beswick, A.D., et al. (2016) Trajectories of Pain and Function after Primary Hip and Knee Artroplasty: The ADAPT Cohort Study. PLoS ONE, 11, e0149306. https://doi.org/10.1371/journal.pone.0149306

[16] Phillips, J.R.A., Hopwood, B., Arthur, C., Stroud, R. and Toms, A.D. (2014) The Natural History of Pain and Neuropathic Pain after Knee Replacement: A Prospective Cohort Study of the Point Prevalence of Pain and Neuropathic Pain to a Minimum Three-Year Follow-Up. Bone \& Joint Journal, 96-B, 1227-1233. https://doi.org/10.1302/0301-620X.96B9.33756

[17] Shipton, E.A. and Tait, B. (2005) Flagging the Pain: Preventing the Burden of Chronic Pain by Identifying and Treating Risk Factors in Acute Pain. European Journal of Anaesthesiology, 22, 405-412. https://doi.org/10.1017/S0265021505000694

[18] Werner, M.U. and Kongsgaard, U.E. (2014) Defining Persistent Post-Surgical Pain: Is an Update Required? British Journal Anaestheesia, 113, 1-4.

https://doi.org/10.1093/bja/aeu012

[19] Li, J.W., Ma, Y.S. and Xiao, L.K. (2019) Postoperative Pain Management in Total Knee Arthroplasty. Orthopaedic Surgery, 11, 755-761.

https://doi.org/10.1111/os.12535

[20] Wylde, V., Beswick, A., Bruce, J., Blom, A., Howells, N. and Gooberman-Hill, R. (2018) Chronic Pain after Total Knee Arthroplasty. EFORT Open Reviews, 3, 461-470. https://doi.org/10.1302/2058-5241.3.180004

[21] Pinto, P.R., McIntyre, T., Ferrero, R., Araujo-Soares, V. and Almeida, A. (2013) Persistent Pain after Total Knee or Hip Arthroplasty: Differential Study of Prevalence, Nature, and Impact. Journal of Pain Research, 6, 691-703. https://doi.org/10.2147/JPR.S45827 
[22] Singh, J.A., Gabriel, S. and Lewallen, D. (2008) The Impact of Gender, Age, and Preoperative Pain Severity on Pain after TKA. Clinical Orthopaedics and Related Research, 466, 2717-2723. https://doi.org/10.1007/s11999-008-0399-9

[23] Meissner, W., Huygen, F., Neugebauer, E.A.M., Osterbrink, J., Benhamou, D., Betteridge, N., et al. (2018) Management of Acute Pain in the Postoperative Setting: The Importance of Quality Indicators. Current Medical Research and Opinion, 34, 187-196. https://doi.org/10.1080/03007995.2017.1391081

[24] Richebe, P., Capdevila, X. and Rivat, C. (2018) Persistent Postsurgical Pain: Pathophysiology and Preventive Pharmacologic Considerations. Anesthesiology, 129, 590-607. https://doi.org/10.1097/ALN.0000000000002238

[25] Ladha, K.S., Patorno, E., Liu, J. and Bateman, B.T. (2016) Impact of Perioperative Epidural Placement on Post Discharge Opioid Use in Patients Undergoing Abdominal Surgery. Anesthesiology, 124, 396-403. https://doi.org/10.1097/ALN.0000000000000952

[26] Ritter, M.A., Wing, J.T., Berend, M.E., Davis, K.E. and Meding, J.B. (2008) The Clinical Effect of Gender on Outcome of Total Knee Arthroplasty. Journal of Arthroplasty, 23, 331-336. https://doi.org/10.1016/j.arth.2007.10.031

[27] Petterson, S.C., Raisis, L., Bodenstab, A. and Snyder-Mackler, L. (2007) Disease-Specific Gender Differences among Total Knee Arthroplasty Candidates. The Journal of Bone \& Joint Surgery, 89, 2327-2333. https://doi.org/10.2106/00004623-200711000-00001

[28] Lim, J.B.T., Chi, C.H., Lo, L.E., Lo, W.T., Chia, S.L., Yeo, S.J., et al. (2015) Gender Difference in Outcome after Total Knee Replacement. Journal of Orthopaedic Surgery, 23, 194-197. https://doi.org/10.1177/230949901502300216

[29] Roth, M.L., Tripp, D.A., Harrison, M.H., Sullivan, M. and Carson, P. (2007) Demographic and Psychosocial Predictors of Acute Perioperative Pain for Total Knee Arthroplasty. Pain Research and Management, 12, Article ID: 394960. https://doi.org/10.1155/2007/394960

[30] Jung, B.F., Gretchen, M.A., Oaklander, A.L. and Dworkin R.W. (2003) Neuropathic Pain Following Breast Cancer Surgery: Proposed Classification and Research Update. Pain, 104, 1-13. https://doi.org/10.1016/S0304-3959(03)00241-0

[31] Drosos, G.I., Triantafilidou, T., Ververidis, A., Agelopoulou, C., Vogiatzaki, T. and Kazakos, K. (2015) Persistent Post-Surgical Pain and Neuropathic Pain after Total Knee Replacement. World Journal of Orthopedics, 6, 528-536. https://doi.org/10.5312/wjo.v6.i7.528

[32] Schofield, P. (2007) Pain in Older Adults: Epidemiology, Impact and Barriers to Management. Reviews in Pain, 1, 12-14.

https://doi.org/10.1177/204946370700100104 


\section{Appendix}

QUESTIONNAIRE

PREVALENCE OF PERSISTENT POST-SURGICAL PAIN IN A TERTIARY CARE CENTRE: A CROSS SECTIONAL SURVEY

$\begin{array}{ll}\text { Date } & \text { MR\# } \\ \text { Age: } & \\ \text { Gender: Male } \square & \text { Female } \square \\ \text { Weight: } \\ \text { Contact \#: }\end{array}$

Anaesthesia:

GA $\square \quad$ Regional

Duration of Surgery:

Intra-operative Analgesia:

Epidural

Paracetamol

I.V Opioids

NSAIDs

Pain score at discharge from recovery room:

0 10

Post-operative Analgesia:

Epidural $\square$

PCIA

Opioid infusion

Intermittent bolus

PRN opioids

Duration of Analgesia (epidural/PCIA etc.):

Co-analgesia:

Paracetamol $\square$

NSAIDs

LA infiltration

Nerve Block

Tramadol

Pain management by APMS

Yes $\square$

No

Satisfaction with postoperative pain management:
1) Very dissatisfied
2) Dissatisfied
3) Neutral
4) Satisfied
5) Very satisfied

Three Months Later

Persistent postoperative pain (PPP) 
Yes $\square$

No $\square$

Area of Pain:

Site of surgery

Surrounding area

Away from surgical site

Type of Pain

Aching $\square$

Burning $\square$

Stabbing

Throbbing $\square$

Degree of Pain (Verbal NRS)

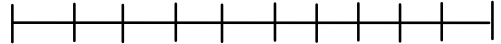

$\begin{array}{lllllllllll}0 & 1 & 2 & 3 & 4 & 5 & 6 & 7 & 8 & 9 & 10\end{array}$

\section{Regarding Pain}

Painful at all-time

Painful on touch

Limited to a single area

Extends to other areas

Painful on movement $\square$

Painful at rest

Odd (altered) sensation on touch

No sensation on touch

Other characteristics

Does the pain disturb sleep?

Yes

No

Does the pain disrupt daily life?

Yes

No

What treatment has the patient received for this pain? 